\title{
THE POSITION AND ORIENTATION MEASUREMENT OF GONDOLA USING A VISUAL CAMERA
}

\author{
$\underline{\text { Hwadong Sun }}^{1}$, Dong Yeop Kim ${ }^{1}$, Joon Ho Kwon ${ }^{2}$, Bong-Seok Kim ${ }^{1}$, and Chang-Woo Park ${ }^{1}$ \\ ${ }^{1}$ Intelligent Robotics Research Center, Korea Electronics Technology Institute, Seoul, Korea \\ ${ }^{2}$ Department of Mechanical Engineering, Korea University, Seoul, Korea \\ * Corresponding author (sword32@keti.re.kr)
}

\begin{abstract}
The research of automation and robotics in building construction industry could improve working conditions through applications which create safety hazards for humans. In this paper, we propose an approach to sense a gondola robot system using a vision camera. To control a gondola safely and accurately, some parameters should be measured such as translation in $\mathrm{X}, \mathrm{Y}$ and $\mathrm{Z}$ axis and rotation (Euler angles) of the gondola. Our approach conducts this operation with inexpensive and easy-to-maintain system.
\end{abstract}

Keywords: Gondola Robot, Building Facade, Balance, Rope

\section{INTRODUCTION}

We make an attempt to maintain building walls with robot system because of following reasons. First, wall maintenance conducted by human is very dangerous. A worker relies only on a piece of rope during end-to-end of building height. When wind speed exceeds the standard, many regulations and laws prohibit working because gust of wind pushes the person on the single wire. The wall maintenance robot can take place of this dangerous job. Second, building wall maintenance requires skillful and expensive workers. The robot system only needs monitoring operators. Their simple jobs are just surveillance about cleaning and maneuver to emergency (maybe pressing stop button).

There are two category of the building wall maintenance robot system. One is built-in type robots [1]. They run through rail on building walls. Therefore, their movements are well-supported. Small sensors are required. However, architects should consider installation of rail on building walls. It may interrupt their design concept of building. The other is gondola type robots [2]. With some mechanical support, this type can be set up at any rooftop. A business model that robots are taken to anywhere customers request is possible. On the other hand, because this type requires a bunch of wire rope to support its weight, it can not be applied to high rise building.

We concentrate on the gondola type robot system. It has some technical problems. First, the localization of the robot is required. Second, the robot should sense and control the balance of body. Third, the gondola should keep contact to wall.

In this paper, we proposed methodology of balance sensing of gondola type robot system using vision system. The rope attribution receives our attention as the key. We put a two dimensional chessboard patched on a box through which the wire rope of gondola goes, and get six degree of freedom (6 DoF) information through vision system. At chapter 2, some preliminaries are introduced. Core description about our solution is suggested at chapter 3 . Chapter 4 shows some experiments. Finally, chapter 5 concludes this paper.

\section{PRELIMINARIES}

A. Six degree of freedom (6 DoF)

In order to describe translation and orientation of a rigid body, six numbers are used. First three numbers are $x, y, z$. They are translation in Cartesian coordinate. Last three numbers are $\alpha, \beta, \gamma$ which are roll, pitch, and yaw, respectively. Roll spins around $x$ axis; pitch is applied to 
$y$, and yaw is for $z$. With these six numbers, we can describe any movement in three-dimensional space.

\section{B. Two dimensional chessboard}

Chessboard is a well known structure for camera calibration[3][4] and we used it to get the box pose. The chessboard corners just happen to be particularly easy to find. The points on a plane undergo perspective transform when viewed through a pinhole or lens. The parameters for this transform are contained in a 3-by-3 homography matrix which contains rotation and translation information. The homography matrix is simply expressed as:

$$
\tilde{q}=s H Q \bar{Q}
$$

Here $\mathrm{q}$ and $\mathrm{Q}$ are a point in an image plane and an object plane in the real world respectively. The parameter $\mathrm{s}$ is an arbitrary scale factor. It is conventionally factored out of $\mathrm{H}$, which is homography matrix.

\section{VISION-BASED 6 DoF EXTRACTING SYSTEM}

\section{A. System overview}

Our goal in this paper is to propose a gondola type robot system that senses its balance using vision system. Fig. 1 is the concept drawing of this approach. We put a box pierced by wire rope and place a 2D barcode on it; a visual camera views this box. A passive arm supports the box.

As the robot system moves, the wire rope follows the movement. Because the rope pierces the box, the box also changes its 6 DoF. We assume that the wire rope acts like stiff body, because the robot system is heavy and the operation should be conducted when wind speed is below a reference value. In inverse order, through finding out the 6 DoF of the box, we can attain the movement of the wall maintenance robot system, and sense its balance.

We already recognized that inertial measurement unit (IMU) sensors do the same things. However, contrast to IMU sensors (e.g. $3 \mathrm{DM}^{1}$ ), this approach has following merits. First, this vision-based system is cheaper than IMU sensors. Since internet webcams were commercialized, the price of $\mathrm{CCD} / \mathrm{CMOS}$ camera has decreased. Second, this vision-based system is placed at rope cart, not at the robot system. It means that operators can take care of the system anytime they want, even though the robot is at middle of the building. It gives convenience for reacting emergency or replacing new system parts. Accordingly, it does not require communicating between rope cart and robot system. Third, fewer disturbances harm sensor outputs than gyroapplied sensor system. Gyro systems depend on geomagnetism and heavy equipments or robot system distort magnetic field around them; distorted gyro output arises. On the other hand, vision system is not affected by magnetic flow. Finally, this vision-based system offers 6 DoF, but gyro system provides only orientation (roll, pitch, and yaw). Additionally, vision-based system extracts 6 DoF on real time. Fig 1. shows our proposed concept.

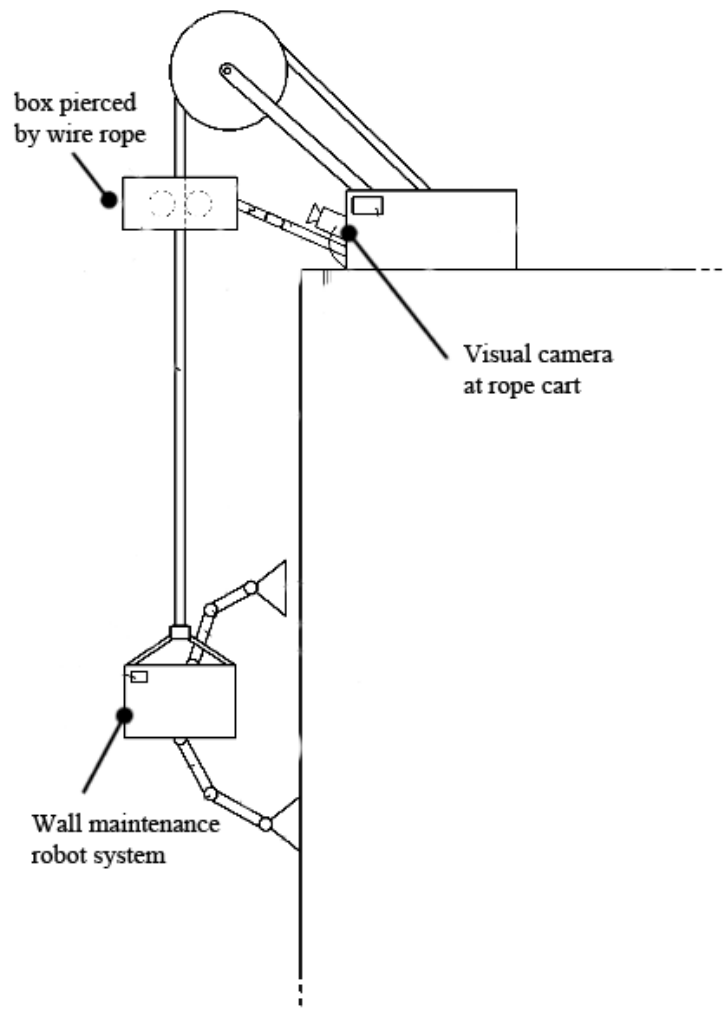

Fig. 1 Tghhe concept of vision sensing system for balance of the wall maintenance robot.

\footnotetext{
${ }^{1}$ http://www.microstrain.com
} 
$\mathrm{H}$ is equal to the camera intrinsic matrix $\mathrm{M}$ multiplied by a combination of the first two rotation matrix columns, $r_{1}$ and $r_{2}$, and the translation vector $\mathrm{t}$.

$$
H=\left[\begin{array}{lll}
h_{1} & h_{2} & h_{3}
\end{array}\right]=s M\left[\begin{array}{lll}
r_{1} & r_{2} & t
\end{array}\right]
$$

Here,

$$
M=\left[\begin{array}{ccc}
f_{x} & 0 & c_{x} \\
0 & f_{y} & c_{y} \\
0 & 0 & 1
\end{array}\right]
$$

Also, it is expressed as:

$$
\begin{array}{r}
r_{1}=\lambda M^{-1} h_{1} \\
r_{2}=\lambda M^{-1} h_{2} \\
r_{3}=\lambda M^{-1} h_{3} \quad(3-3)
\end{array}
$$

Here, $\lambda=1 / \varepsilon$.

Algebraically, a rotation matrix in n-dimensions is a n-by-n special orthogonal matrix. Using the fact, we can get $r_{3}$.

$$
r_{3}=r_{1} \times r_{2}
$$

To get a rotation matrix and translation matrix, we need to get $\mathrm{M}$ and $\mathrm{X}$.

Using the knowledge that $r_{1}$ and $r_{2}$ are orthonormal, the first constraint is derived as:

$$
\begin{aligned}
& r_{1}^{T} r_{2}=0 \\
& \left(\lambda M^{-1} h_{1}\right)^{T}\left(\lambda M^{-1} h_{2}\right)=0 \\
& h_{1}^{T} M^{-T} M^{-1} h_{2}=0
\end{aligned}
$$

Here, $\mathrm{M}^{\mathbf{T}}$ is shorthand for $\left(\mathrm{M}^{-4}\right) \mathrm{T}$.

The second constraint could defined by the fact that the magnitudes of the rotation vectors are equal.

$$
\begin{aligned}
& \left\|r_{1}\right\|=\left\|r_{2}\right\| \\
& r_{1}^{T} r_{1}=r_{2}^{T} r_{2} \\
& h_{1}^{T} M^{-T} M^{-1} h_{1}=h_{2}^{T} M^{-T} M^{-1} h_{2}
\end{aligned}
$$

$$
B=M^{-T} M^{-1}=\left[\begin{array}{lll}
B_{11} & B_{12} & B_{13} \\
B_{21} & B_{22} & B_{23} \\
B_{31} & B_{32} & B_{33}
\end{array}\right]
$$

$$
=\left[\begin{array}{ccc}
\frac{1}{f_{x}^{2}} & 0 & \frac{-c_{x}}{f_{x}^{2}} \\
0 & \frac{1}{f_{y}^{2}} & \frac{-c_{y}}{f_{y}^{2}} \\
\frac{-c_{x}}{f_{x}^{2}} & \frac{-c_{y}}{f_{y}^{2}} & \frac{c_{x}^{2}}{f_{x}^{2}}+\frac{c_{y}^{2}}{f_{y}^{2}}+1
\end{array}\right]
$$

Two constraints have the general form.

$$
\begin{aligned}
& \mathrm{h}_{\mathrm{j}}^{\mathrm{T}} \mathrm{Bh}_{\mathrm{j}} \\
& \begin{aligned}
=\left(\mathrm{h}_{\mathrm{i} 1} B_{11}+\mathrm{h}_{\mathrm{i} 2} B_{21}\right. & \left.+\mathrm{h}_{\mathrm{i} 3} B_{31}\right) h_{\mathrm{j} 1} \\
& +\left(\mathrm{h}_{\mathrm{i} 1} B_{12}+\mathrm{h}_{\mathrm{i} 2} B_{22}+\mathrm{h}_{\mathrm{i3}} B_{32}\right) h_{\mathrm{j} 2} \\
& +\left(\mathrm{h}_{\mathrm{i} 1} B_{13}+\mathrm{h}_{\mathrm{i} 2} B_{23}+\mathrm{h}_{\mathrm{i} 3} B_{33}\right) h_{\mathrm{j} 3}
\end{aligned}
\end{aligned}
$$

$$
\begin{aligned}
=h_{i 1} B_{11} h_{j 1}+h_{i 2} B_{21} h_{j 1}+h_{i 3} B_{31} h_{j 1}+h_{i 1} B_{12} h_{j 2} \\
+h_{i 2} B_{22} h_{j 2}+h_{i 3} B_{32} h_{j 2}+h_{i 1} B_{13} h_{j 3} \\
+h_{i 2} B_{23} h_{j 3}+h_{i 3} B_{33} h_{j 3}
\end{aligned}
$$

$$
\begin{aligned}
& \therefore \text { i is symmetric, } B_{12}=B_{21}, B_{13}=B_{31}, B_{23}=B_{32} \\
& \begin{aligned}
=h_{i 1} h_{j 1} B_{11}+( & \left.h_{i 1} h_{j 2}+h_{i 2} h_{j 1}\right) B_{12}+h_{i 2} h_{j 2} B_{22} \\
+ & \left(h_{i 3} h_{j 1}+h_{i 1} h_{j 3}\right) B_{13} \\
& +\left(h_{i 3} h_{j 2}+h_{i 2} h_{j 3}\right) B_{23}+h_{i 3} h_{j 3} B_{33}
\end{aligned}
\end{aligned}
$$

$$
=\left[\begin{array}{c}
h_{i 1} h_{j 1} \\
h_{i 1} h_{j 2}+h_{i 2} h_{j 1} \\
h_{i 2} h_{j 2} \\
h_{i 3} h_{j 1}+h_{i 1} h_{j 3} \\
h_{i 3} h_{j 2}+h_{i 2} h_{j 3} \\
h_{i 3} h_{j 3}
\end{array}\right]^{T}\left[\begin{array}{c}
B_{11} \\
B_{12} \\
B_{22} \\
B_{13} \\
B_{23} \\
B_{33}
\end{array}\right]=v_{i j}^{T}
$$

Using ${v_{i j}}^{\mathrm{T}}$, two constraints which are (3-5), (3-6) may written as the following equation.

$$
\left[\begin{array}{c}
v_{12}^{\mathrm{T}} \\
\left(v_{11}-v_{22}\right)^{\mathrm{T}}
\end{array}\right] \mathrm{b}=0
$$

Let 
If $\mathrm{n}$ images of the chessboard are observed, by stacking $\mathrm{n}$ such equations as (3-9) we have a follow equation.

$$
\mathrm{Vb}=0
$$

Here, $\mathrm{V}$ is a $2 \mathrm{n}-\mathrm{by}-6$ matrix. If $\mathrm{m} \mathrm{z}$, a unique solution $\mathrm{b}$ defined up to a scale factor is obtainable. The equation (310) is well known as the eigenvector of $x^{\mathrm{T}} \mathrm{p}$ associated with the smallest eigenvalue.

\section{EXPERIMENT}

Fig. 2 shows the experiment environment where we used wire rope applied for the construction field, a webcam(CMOS camera), and a laptop. The box is moving continuously and grabbed some scene by the camera. Fig. 2 shows the Experiment environment.

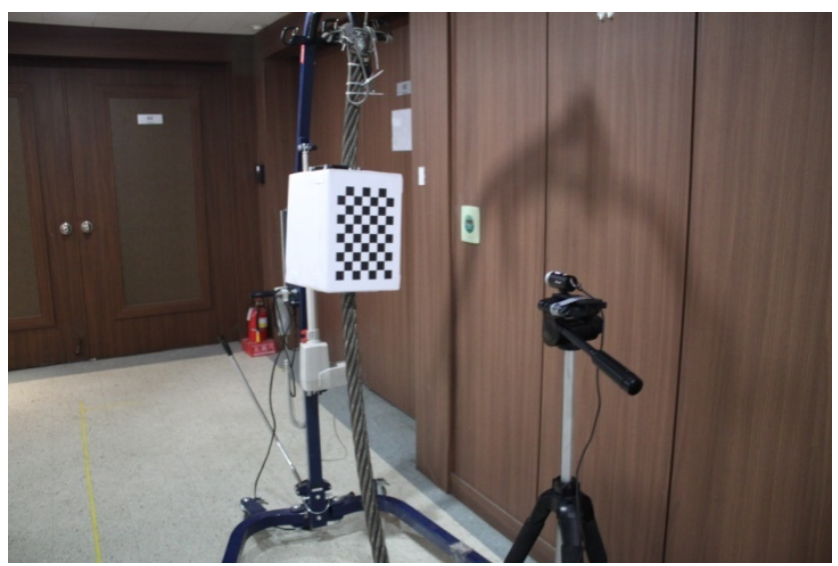

Fig. 2 Experiment environment

45 interior corners are on the board. Also, the square boxes of the chessboard have same size which is known. Fig. 3 shows the result of corner detection.

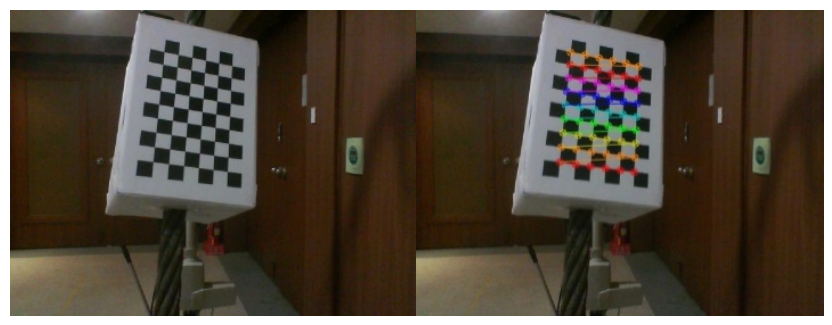

(a) Raw Image

(b) Chessboard corners
Every rotation result of each scene is compared by gyro results. Fig. 4 shows the comparison.

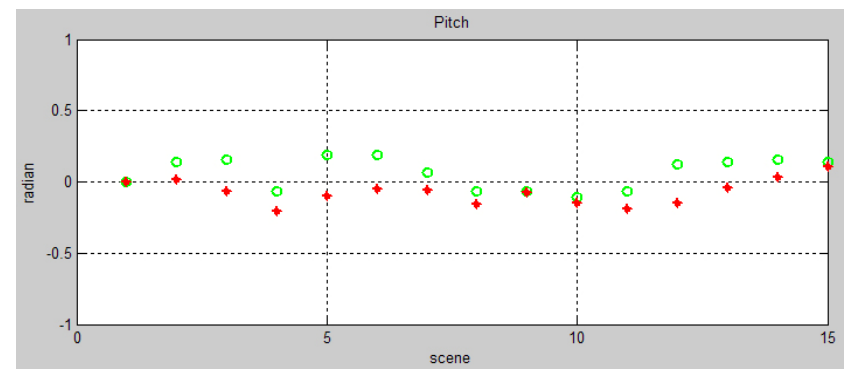

(a) Roll

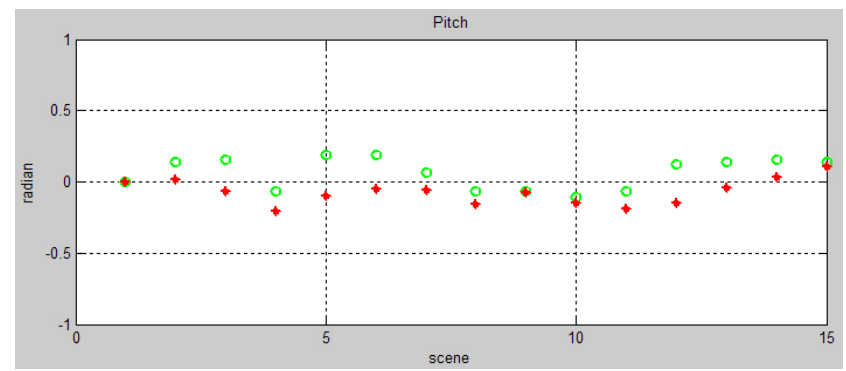

(b) Pitch

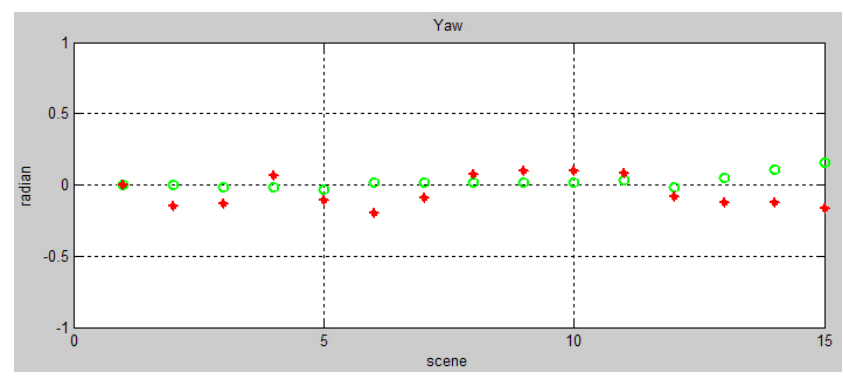

(c) Yaw

Fig. 4 Comparison of Euler angles between gyro (green) and the result by camera (red)

We got fifteen samples, and extracted their orientations. Yaxis of Fig. 4 is the gap between first sample and current sample. The reason we use the gap is in order to guarantee coincide of coordinate. For example, the fourth value of Fig. 4 is the gap of orientation between fourth sample and first sample.

Also, Fig. 5 shows the error graph. The error means the difference between gyro and the result by camera. 


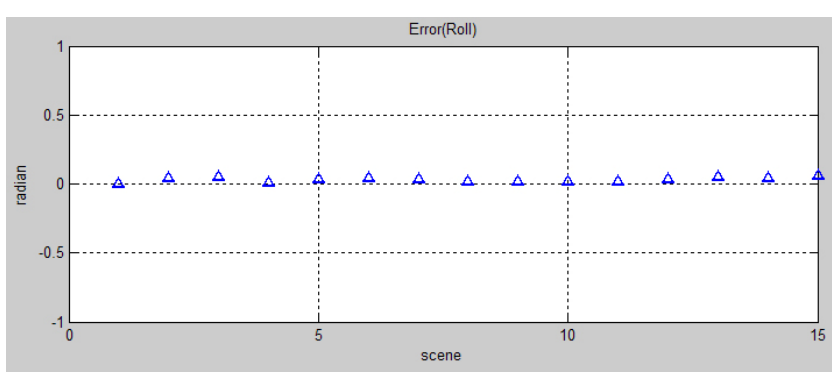

(a) Roll

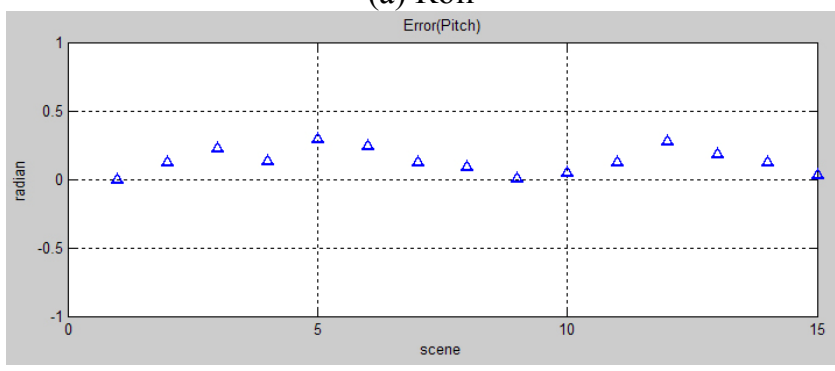

(b) Pitch

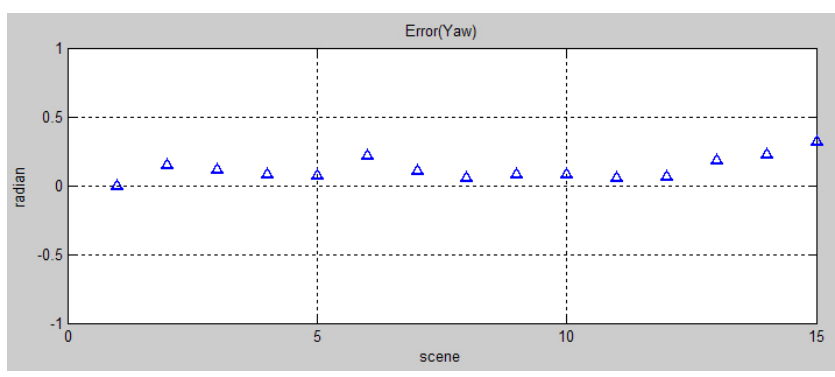

(c) Yaw

Fig. 5 Error values

\section{CONCLUSION}

We proposed a sensor system for gondola type building maintenance robot. Main jobs of the robot system are painting, cleaning, and repairing. Therefore, sensing and controlling balance of the robot is important.

Our system is cheaper than an IMU sensor system; easy to maintenance; robust to magnetic noise. Additionally, it provides 6 DoF (i.e. translation and rotation).

In future, we will get other sensing systems. Then, sensor fusion will be applied.

\section{ACKNOWLEDGMENTS}

The work presented in this paper was funded by BMRC(Building-Façade Maintenance Robot Research Center), supported by Korea Institute of Construction and Transportation Technology Evaluation and Planning (KICTEP) under the Ministry of Land, Transport and Maritime Affairs(MLTM).

\section{REFERENCES}

[1] M. Sack, N. Elkmann, T. Felsch, and T. Bohme, "Intelligent control of modular kinematics - the robot platform STRIUS", in Proc. of the 2002 IEEE International Symposium on Intelligent Control, 2002, pp. $549-553$

[2] H. Zhang, J. Zhang, and G. Zong, "Requirements of glass cleaning and development of climbing robot systems", in Proc. of the 2004 International Conference on Intelligent Mechatronics and Automation, Aug. 2004, pp. 101-106.

[3] R. Zhang, P.-S. Tsi, J. E. Cryer, and M. Shah, "Shape form shading: A survey," IEEE Transactions on Pattern Analysis and Machine Intelligence 21 (1999): 690-706.

[4] Z. Zhang, "A flexible new technique for camera calibration," IEEE Transactions on Pattern Analysis and Machine Intelligence 22 (2000): 1330-1334.

[5] Sutheebanjard, P and Premchaiswadi, W., "QR-code generator", in Proc. of 2010 Eighth International Conference on ICT and Knowledge Engineering, Nov. 2010, pp. 89-92.

[6] J. Wang, C. Shyi, and T.-W. Hou, and C.P. Fong, "Design and implementation of augmented reality system collaborating with QR code", in Proc. of 2010 International Computer Symposium (ICS), Dec. 2010, pp. 414-418. 